\title{
Design Principles and Improvement of the Evaluation Methods for the Shatter Strength of Metallurgical Materials
}

\author{
Wendan Tang ${ }^{1,}$, , Junxue Zhao ${ }^{1}$, Liang Niu' ${ }^{1}$, Lv Zhao ${ }^{2}$, Tingliang Zhang ${ }^{2}$ \\ ${ }^{1}$ College of Metallurgy Engineering, Xi' an University of Architecture and Technology, Xi' an, China \\ ${ }^{2}$ Hanzhong Zinc Industry Co., Ltd., Hanzhong, China
}

Email address:

tangba8601@foxmail.com (Wendan Tang)

${ }^{*}$ Corresponding author

\section{To cite this article:}

Wendan Tang, Junxue Zhao, Liang Niu, Lv Zhao, Tingliang Zhang. Design Principles and Improvement of the Evaluation Methods for the Shatter Strength of Metallurgical Materials. International Journal of Mineral Processing and Extractive Metallurgy.

Vol. 6, No. 2, 2021, pp. 24-32. doi: 10.11648/j.ijmpem.20210602.12

Received: April 6, 2021; Accepted: May 15, 2021; Published: May 31, 2021

\begin{abstract}
Shatter strength refers to the ability of materials to resist collisions and impacts and is an important property of metallurgical minerals. Although the current methods differ in terms of the testing equipment, implementation steps and data processing methods, these approaches are nearly identical in principle. However, the current methods are not sufficiently accurate, which makes it difficult to objectively evaluate shatter strength. Therefore, new and more accurate methods are needed. In this paper, a variety of current methods were discussed, design principles were summarized, and new methods were proposed that consider the influence of the degree of rupture and drop time when cracks are generated on the results of shatter strength tests. Based on the new design principles, new parameters such as the crack size index, number of fragments, and total mass of the fragments were added to the evaluation formula. In addition, the test processes were optimized. Then, a batch of lead-containing pellets were evaluated by the new methods. The results obtained in this test show that, compared with the old methods, the new methods can more accurately and objectively evaluate shatter strength and reflect product quality. Users can also design new methods for all kinds of brittle materials according to these principles. However, the new method is more complex than the old methods and needs to consider a greater number of factors. At present, there is no effective means to address the workload. With the development of artificial intelligence and automation devices, new design principles and methods will be more widely utilized.
\end{abstract}

Keywords: Shatter/Drop/Falling Strength, Evaluation Method, Metallurgical Material

\section{Introduction}

Shatter strength refers to the ability of materials to resist collisions and impacts, which is an important property of metallurgical materials. In the actual testing process, the shatter strength is defined as either the number of cracks generated when a material drops from a certain height and hits a specific steel plate or the proportion of material quality remaining after a certain number of drops. In practical applications, good shatter strength generally means that the materials do not shatter or deform during the processes of transportation and burden distribution in a furnace where they are melted to make slag by means of high-temperature smelting without producing much dust or steam [1-2]. For researchers, the shatter strength evaluation method is an important means to study the crushing and impact resistance of metallurgical materials. In the market, shatter strength is an important basis for price negotiation.

Shatter strength evaluations can be applied to ferrous (e.g., sinter, green pellets, pellets, coke, and briquettes) and nonferrous metallurgical materials (beneficiated burden materials used in copper, lead and zinc smelting processes, including pellets or other forms of particles) [1-9]. There are corresponding national standards for different ferrous metallurgical materials, coke and coal but no standards for nonferrous metallurgical materials and other minerals. Owing to the physical properties and conditions of use of different ferrous metallurgical materials, there are certain differences in testing methods. Various countries and institutions may have 
different testing standards for the same material [10-18]. These differences are reflected mainly in the testing equipment, execution steps and data processing methods, as shown in Table 1. These evaluation methods are nearly identical in principle, but they are not highly accurate. With the current approaches, it is difficult to objectively evaluate the ability of a material to resist collisions and impacts. Thus, a new method should be developed for more effective testing. This text will compare different evaluation methods and then propose a new evaluation method along with its design principle, which offers a wider application scope and better accuracy.

\section{Discussion of Evaluation Methods for the Shatter Strength of Different Materials}

\subsection{Differences in Evaluation Methods Between Green Pellets and Other Materials}

Table 1 clearly shows that there are two main differences between green pellets and other materials $[1,2]$ : the selection of drop height and evaluation value $N$. The drop height of the green pellets is $0.5 \mathrm{~m}$, whereas that for all other metallurgical materials is greater than $1.8 \mathrm{~m}$. The evaluation value $N$ of the green pellets is the drop time when cracking occurs, whereas the evaluation value $n$ for the other materials is the percentage of residual metallurgical material.

The reason for the difference in drop height is that the drop height of the green pellets in the transfer process is approximately $0.5 \mathrm{~m}$. Thus, if the drop height of green pellets is too large, the test condition does not coincide with the actual situation, and the test accuracy is affected. For the same reason, the drop heights of other metallurgical materials are between $\sim 1.8$ and $2 \mathrm{~m}$.

The main reason for the difference in the selection of evaluation values $N$ is that it is easy to observe cracks in green pellets. However, other materials, such as coal or sinter, are porous, hard and brittle, which makes it difficult to observe cracks. Thus, for other materials, evaluating the percentage of residual material is the better option.

In addition, there are some differences in the evaluation procedures. The shatter strength of green pellets is marked by the appearance of cracks during the test. If cracks do not appear, the test cannot be finished. However, the shatter strength of other materials can be calculated after a fixed drop time. Hence, the test of other materials will definitely be finished.

\subsection{Testing Equipment and Data Processing Methods}

For green pellets, the traditional methods can be performed by hand or using testing equipment that can contain 100-200 $6 \sim 20 \mathrm{~mm}$ samples $[1,8,10]$. The testing equipment performs an automatic dropping process; this automation reduces labour. However, it is still necessary to manually observe the time when the pellets crack or break. The special equipment designed by Prof. Li Jiaxin allows the pellets to drop one by one, which is more efficient. When this equipment is used, the testing speed can be increased by more than $50 \%$. However, the pellets may collide with each other, causing additional inaccuracies. In addition, the data processing method proposed by Prof. Li provides more accurate results than the traditional method [8, 10]. This evaluation method is no longer limited to the average drop time for multiple samples but simultaneously measures the drop times $i$ and the number of cracked samples $m_{i}$ after each drop. Greater values of $i$ and $m_{i}$ correspond to a greater weight, and the final value $n$ conforms to the statistical rule. However, method requires a larger number of samples than the traditional method, to make the test data converge.

Other metallurgical material evaluation methods require a total sample mass of approximately $6-20 \mathrm{~kg}$ for each test; thus, testing equipment must be used [8, 10-20]. Each testing device consists of a sample box and a lifting device. Prototype equipment first appeared in Soviet-era Russian standards for testing sinter, iron ore and green pellets. Subsequently, this equipment became more sophisticated and diversified, after which it was used for testing coke, briquettes and other materials. During testing, standard-size samples were placed in the sample box, which was raised to a predetermined height and then dropped. Each sample was dropped 3-4 times, and three aliquots were collected. However, during the use of this testing method, it was found that the results of individual tests were quite different. A study found that these evaluation methods ignored statistics for the process of each drop and recorded only the final results [19-20]. Therefore, it is meaningful to design a new method based on previous work and include process statistics. Setting design principles based on accuracy and applicability facilitates the establishment of new methods.

\subsection{Thoughts on Improving the Current Testing Methods and Principle Setting}

Industry and national standards are usually applied to obtain test results quickly and easily [5], enabling buyers and sellers to quickly reach a deal. The researchers hope to obtain accurate results. Therefore, convenient, accurate and adaptable methods have a wide range of requirements. The current testing methods have the following detailed problems:

For green pellets, the first crack in different samples may not appear at the same time, and the crack size may also be different; therefore, the results should be different. However, the current methods consider these results equal.

Current methods lack a means to evaluate the dropping process. For the coal test, one sample may break more in the first drop and less in the second drop, whereas another sample may break less in the first drop and more in the second drop. However, if the final percentage of residual material is the same, then the current methods consider these results to be equivalent. This can lead to the same results for various batches of samples, but the data distribution from repeated tests is likely to be different; thus, the results should differ.

Based on the above discussion, the underlying principle of the new method is summarized as follows:

Shatter strength of green pellets

Principle a: Samples with cracks first $<$ samples with cracks 
later.

Principle b: When cracks occur simultaneously: sample with a large crack $<$ sample with a small crack.

Principle c: When the final values are the same: sample with larger data discreteness $<$ sample with smaller data discreteness.

Shatter strength of other materials

Principle A: $1^{\text {st }}$ broken sample $<2^{\text {nd }}$ broken sample $<3^{\text {rd }}$, etc.

Principle B: Samples with more fragments $<$ samples with fewer fragments.

Principle C: Sample with a small residual mass $<$ sample with a large residual mass.

Principle D: When the final value is the same: sample with large data discreteness $<$ sample with small data discreteness.

Table 1. Shatter strength testing methods of different materials.

\begin{tabular}{|c|c|c|c|c|c|c|c|c|}
\hline No. & Years & Equation & $\begin{array}{l}\text { Application } \\
\text { object }\end{array}$ & $\begin{array}{l}\text { Drop } \\
\text { height } / \mathrm{m}\end{array}$ & $\begin{array}{l}\text { Initial } \\
\text { dimension/mm }\end{array}$ & $\begin{array}{l}\text { Dimension at } \\
\text { termination } / \mathrm{mm}\end{array}$ & $\begin{array}{l}\text { Termination } \\
\text { status }\end{array}$ & $\begin{array}{l}\text { Sample quantity per } \\
\text { test }\end{array}$ \\
\hline 1 & $1980 \mathrm{~s}$ & $N$ & Green pellets & 0.5 & $9 \sim 16$ & - & Crack & $N$ (Num.) \\
\hline 2 & 1982 & $n=\frac{M}{M+M_{0}} \times 100 \%$ & $\begin{array}{l}\text { Iron ore, sinter, } \\
\text { pellet }\end{array}$ & 2 & $\begin{array}{l}\text { pellet } 5 \sim 15 \\
\text { iron } 10 \sim 40 \\
\text { sinter } 5 \sim 40\end{array}$ & 5 & Crush & $15 \pm 0.15 \mathrm{~kg}$ \\
\hline 3 & 1993 & $n=\frac{M_{1}}{M_{0}} \times 100 \%$ & $\begin{array}{l}\text { Iron ore and } \\
\text { sinter }\end{array}$ & 2 & $50 \pm 10$ & 10 & Crush & $20 \pm 0.2 \mathrm{~kg}$ \\
\hline 4 & 1997 & $n=\frac{M_{1}}{M_{0}} \times 100 \%$ & Iron ore & 2 & $50 \pm 10$ & 10 & Crush & $20 \pm 0.2 \mathrm{~kg}$ \\
\hline 5 & 1999 & $S I_{4}^{50}(\%)=\frac{G_{1}}{G} \times 100 \%$ & Coke & 1.83 & $>80$ or $>60$ & 50 & Crush & $25 \pm 0.1 \mathrm{~kg}$ \\
\hline 6 & 2004 & $D S(\%)=\frac{M_{1}}{M_{0}} \times 100 \%$ & $\begin{array}{l}\text { Industrial } \\
\text { briquette }\end{array}$ & 2 & $>13$ & 13 & Crush & $6 \mathrm{~kg}$ \\
\hline 7 & 2006 & $S_{25}=\frac{M_{1}}{M_{0}} \times 100 \%$ & Coal & 2 & $60 \sim 100$ & 25 & Crush & 10 (Num.) \\
\hline 8 & 2016 & $n=\frac{\sum m_{i} \times(i-1)}{M}$ & Green pellets & 0.5 & $10 \sim 18$ & - & Crack & $N$ (Num.) \\
\hline 9 & 2017 & $S_{I}=\frac{M_{1}}{M_{0}} \times 100 \%$ & Sinter & 2 & $10 \sim 50$ & 10 & Crush & $20 \sim 50 \mathrm{~kg}$ \\
\hline
\end{tabular}

Table 1. Continued.

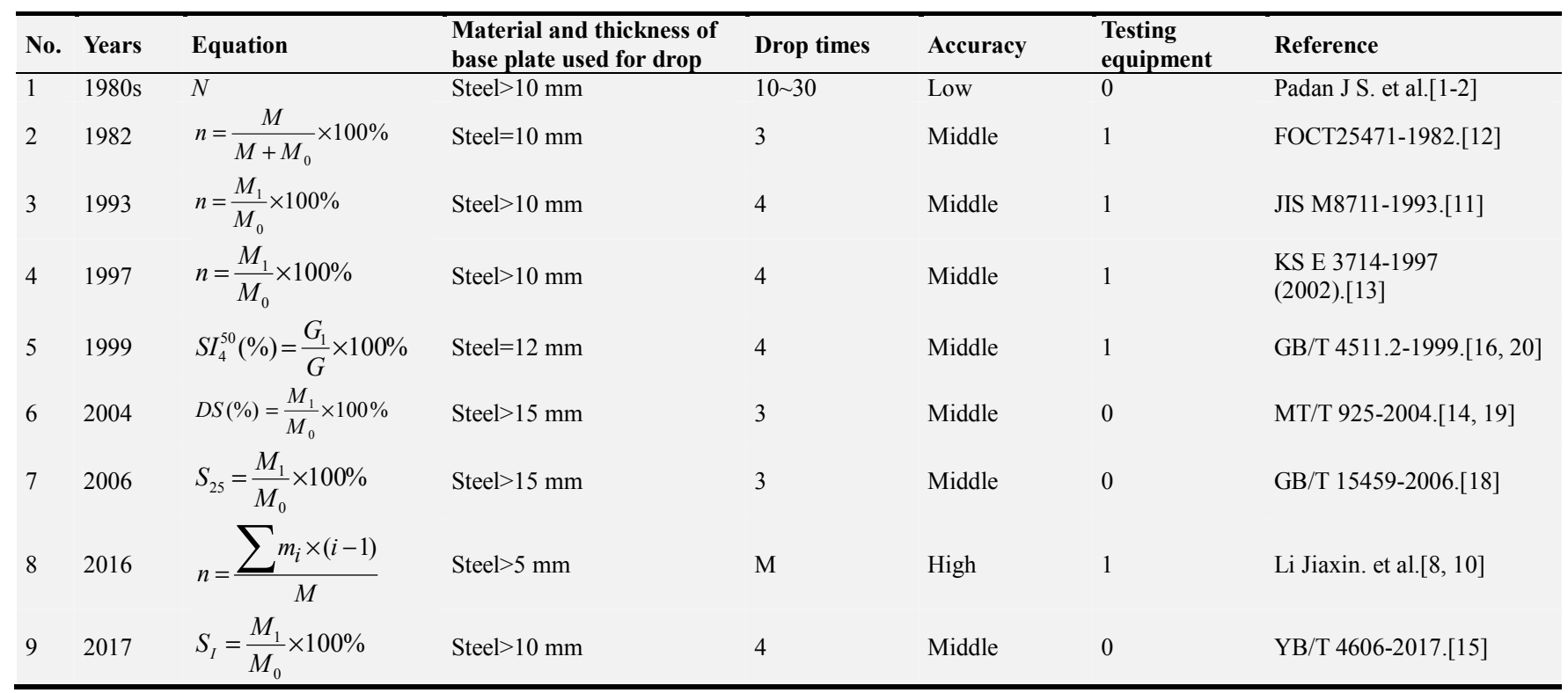

Notes:

1. The numerator is the mass of the sample greater than the termination size, and the denominator is the original total mass.

2. The judgement of test accuracy depends on the error handling method and the accuracy of the formula itself.

3. The drop height of the green pellets is $0.5 \mathrm{~m}$, where $\mathrm{i}$ and $N$ is the drop time, $n$ is the number of samples, $m_{i}$ represents the number of pellets with cracks, and $M$ represents the number of pellets used in the test.

4. In the testing equipment column, 1 means yes and 0 means no.

5. If only the state after the test is considered, the accuracy is low. If the state before the test is additionally considered, middle; if the state before the test and the intermediate process are both considered, high. 
The testing process of the new method is described in Figure 1.

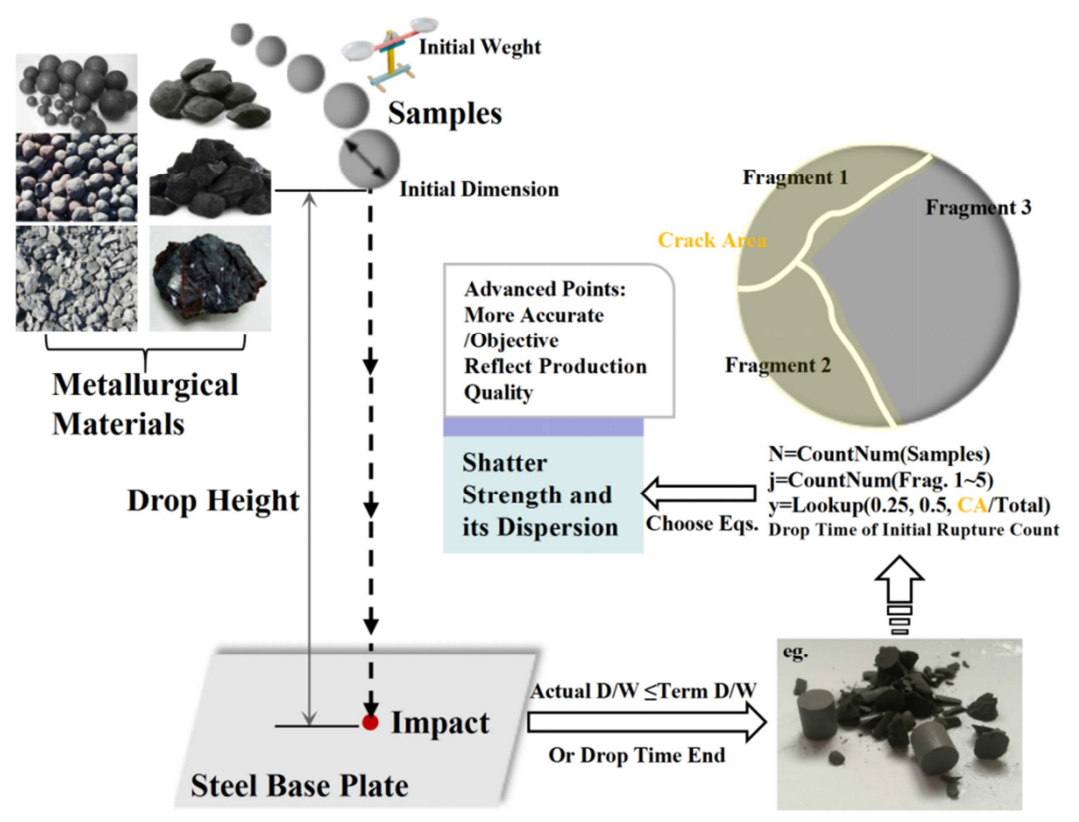

Figure 1. The testing process and the principle of the new method.

\section{New Testing Method and Procedures}

\subsection{Evaluation Method and Procedures for Green Pellet Production}

$$
\begin{gathered}
Q_{L X-i}=\frac{1}{j_{i}} \cdot m_{L-i}^{1 / y_{i}} \\
Q_{L X}=\frac{1}{N} \sum_{i=1}^{N>12} Q_{L X-i}=\bar{Q}_{L X} \\
V_{L X}=\frac{\sqrt{\frac{1}{N-1} \sum_{i=1}^{N>12}\left(Q_{L X-i}-\bar{Q}_{\mathrm{LX}}\right)^{2}}}{\left|\bar{Q}_{\mathrm{LX}}\right|}
\end{gathered}
$$

According to the research of other scientists and the principles set in Section 2.3, the new evaluation method for green pellets is shown in Eqs. 1-3. In these equations, the meanings of the symbols are as follows:

$i$ represents the serial number of test samples;

$Q_{L X-i}$ refers the shatter strength of a sample;

$Q_{L X}$ refers to the final shatter strength of batch samples, which is actually the weighted result of multiple $Q_{L X-i}$;

$\bar{Q}_{L X}$ is the average of $Q_{L X-i}$, which is the same value as $Q_{L X}$;

$V_{L X}$ represents the shatter strength discreteness of a batch of samples, which is used to measure the rationality of the data distribution; Principle c, Principle D

$m_{L-i}$ is the drop time when cracks are generated; Principle $a$.

$N$ is the total number of samples tested, in which $N \geq 12$. Less samples than traditional method can make the test data converge.

$j_{i}$ is the number of fragments, which is used when the green pellets are split, wherein fragments $>20 \%$ original mass are counted ( $j=1$ when the sample does not break into two parts, $j=2$ when the sample breaks into two parts, $j=3$ when the sample breaks into three parts, $j=4$ when the sample breaks into four parts, and $j=5$ if there are no fragments $>20 \%$ of the original mass). Principle $b$.

$1 / y$ is the crack size index, wherein $y=3,2$ and 1 if the percentage of the pellet surface area (including the state of fragmentation) covered by the crack is $>1 / 2,1 / 4 \sim 1 / 2$, and $0 \sim 1 / 4$, respectively. Principle $b$.

Testing process:

a. Random sampling or systematic sampling is used for testing. According to the overall quality of the test batch of metallurgical material, a sufficiently large number of samples is $N \geq 12$. The original mass of each sample is weighed and recorded.

b. The sample is dropped from a height of $0.5 \mathrm{~m}$ (or other required height) onto a carbon steel plate that is thicker than $10 \mathrm{~mm}$. The sample is repeatedly dropped until cracking or rupture occurs.

c. The drop times $m_{L-i}$ of the sample crack or rupture generation, the crack size index $1 / y_{i}$, and the number of fragments $j_{i}$ are recorded.

d. After all samples are tested, the output data are substituted into Eqs. 1 3 to calculate $Q_{L X}$ and $V_{L X} . V_{L X} \leq 1$ is considered qualified. If unqualified, twice the initial number of samples will be used for an additional inspection.

e. If the $Q_{L X}$ values of multiple sample batches are equal, the corresponding values of $V_{L X}$ are compared; if the $V_{L X}$ is smaller, the shatter strength $Q_{L X}$ is larger (original $Q_{L X}+0.01$ ) and vice versa.

\subsection{Evaluation Method and Procedures for Other Materials}

The test samples of green pellets have regular shapes and small sizes, whereas other samples, such as sinter and coke, have 
irregular shapes and large sizes. Thus, each sample has a different minimum quality requirement. The shatter strength of the other material is usually expressed as a percentage of the residual material that meets the dimension requirements. According to the research of other scientists and the principles set in Section 2.4, the calculation of $Q_{L X-i}$ for other materials is shown in Eq. 4. Eqs. 2-3 are also used to calculate the discreteness $V_{L X}$ and the final shatter strength $Q_{L X}$ of these materials.

$$
Q_{L X-i}=\frac{1}{j_{i}} \cdot\left(\frac{m_{L-i}}{m_{0-i}} \times 100\right)^{\frac{x_{i}}{k}}+\left(\frac{x_{i}}{k}-1\right) \times 100
$$

Here, $i$ refers to the serial number of the test sample;

$k$ is the total number of drops selected according to the needs, which is generally $3-4$;

$x_{i}$ is the drop time when the test is terminated (because the sample is completely shattered and cannot continue), in which $x_{i} \leq k ;$ Principle $A$

$m_{0-i}$ is the original mass of the sample;

$m_{L-i}$ is the total mass of the fragments conforming to the standard after the test; Principle $C$

$N$ denotes the number of samples for the test, wherein $N \geq 12$;

$j_{i}$ is the number of fragments with mass $\geqq 20 \% \mathrm{~m}_{0}$ after each drop. When no fragment mass was $\geq 20 \% m_{0}$ after the test, the degree of fragmentation was very large. The total mass of fragments with mass $\geq 10 \% m_{0}$ is recorded, $j_{i}=5$. For certain materials, such as iron ore, coke and coal, a test may require multiple samples, in which case $j_{i}=j_{n}{ }^{\prime} / j_{0}$, wherein $j_{0}$ is the number of samples before testing and $j_{n}$ ' is the number of fragments $>20 \% m_{0-i}$ of the smallest sample after each drop. Principle $B$

Testing procedure:

a. A random sampling method or systematic sampling method should be adopted to take samples from different metallurgical materials according to the corresponding national or industrial standards. The sample size and quality should meet the standard requirements, $N \geq 12$. The $\mathrm{m}_{0 \text {-i }}$ value of each sample is recorded, and then, the sample is placed in an oven to dry at $115 \pm 5^{\circ} \mathrm{C}$ for $12 \mathrm{~h}$.

b. The sample i placed into the sample box, and then, the box is dropped onto a 10 -mm-thick carbon steel plate at the height specified in the corresponding standard. After the first drop, fragments with the required quality $\left(>20 \% m_{0-i}\right)$ are selected for the second drop, and this operation is repeated until the predetermined number of drops $k$ is reached. If the parameter $x$ exists, the corresponding $x_{i}$ value is recorded; if not, $x_{i}$ is taken as equal to $k$, and both $m_{L-i}$ and $j_{i}$ are recorded. Notably, the evaluation of $j_{i}$ usually requires an electronic balance to measure the mass of the pieces $>20 \% m_{0-i}$.

c. The previous item is repeated, and the parameters $x_{i}, m_{L-i}$ and $j_{i}$ are tested

d. The shatter strength $Q_{L X-i}$ of each sample is calculated with Eq. 4 . The average shatter strength $Q_{L X}$ and final shatter strength $Q_{L X}$ of the batch of materials are calculated, and the data discreteness $V_{L X}$ is determined When $V_{L X} \leq 1$, all of the data are qualified; otherwise, twice the initial number of samples will be used for an additional inspection.

e. If the $Q_{L X}$ values of multiple samples are equal, the corresponding values of $V_{L X}$; are compared when the value of $V_{L X}$ is smaller, and the shatter strength $Q_{L X}$ is larger.

The significance of setting the parameter $j$ is to calculate the degree of breakage and is as follows: under the same $m_{L}$ condition, the larger $j$ is, the smaller the shatter strength. The significance of setting the parameter $x$ is to distinguish between samples that can and cannot meet the total number of drops $k$. Under the condition that $k$ is large, the $Q_{L X-i}$ value of the samples that cannot complete the predetermined number of drops will decrease or even become negative. When the discreteness $V_{L X}>1$, the test results may be problematic. If $V_{L X}>1$ remains after retesting with twice the initial number of samples, the quality of the sample batch is not good and may not be suitable for furnaces. The disadvantage of this evaluation method is that the parameter setting, testing and data processing will be more complicated.

\section{Application Example}

Among the methods shown in this paper, the evaluation method of green pellets is generally applied to samples with small particle sizes (less than $20 \mathrm{~mm}$ ), low shatter strength, and visible cracks. The evaluation methods of other materials are generally applied to brittle samples with large particle sizes that easily flake and make crack observation difficult. Except for the drop height and sample weight, the other conditions of the two methods are similar.

The two methods are used to test lead-containing pellets at the same time, which are pelletized and used in the direct smelting process. The pellets are prepared by pressing with the same proportion of materials and different water contents. The crushing resistance values of pellets with different water contents are very different. Particles with a low water content easily crack and crush, whereas particles with high water content have very hard surfaces and strong crushing resistance. The particle size of each pellet was $12 \pm 1 \mathrm{~mm}$, and the weight was $2.6 \pm 0.3 \mathrm{~g}$.

\subsection{Details of the Testing Process}

Because the drop height in the process of particle transport is essentially above $2 \mathrm{~m}$, the drop height is set to $2 \mathrm{~m}$, and other conditions remain unchanged. For the green pellets, the results obtained by the traditional method (Table 1-No. 1) and the new method (Eqs. 1-3) are shown in Table A1 and Table A2, respectively. For the other materials, the results obtained by the traditional method (Table 1-No. 2) and the new method (Eqs. 2-4) are shown in Table A3 and Table A4, respectively; the total number of drops $k$ obtained by the traditional and new methods is 3 and 8 , respectively.

\subsection{Results and Analysis}

The relationship between the test results for the four groups is described hereafter. $Q_{L x-I}$ is obtained by the traditional test 
method for the shatter strength for green pellets (Table 1-No. $1)$, which directly counts the number of broken pellets. For the green pellets, $Q_{L x-2}$ is obtained by Eqs. 1-3, which considers the impact of the degree of rupture. $Q_{L x-3}$ is obtained by following the Table 1-No. 2 method, and it is expressed by the percentage of residual mass after fragmentation. $Q_{L x-4}$ is obtained by Eqs. 2-4 for other materials, and it takes into account the influence of the termination drop times $x$ and the number of fragments $j$ on the final result. $V_{L x-i}$ corresponds to the discreteness of the results $Q_{L X-i}$, as shown in Figure 3.

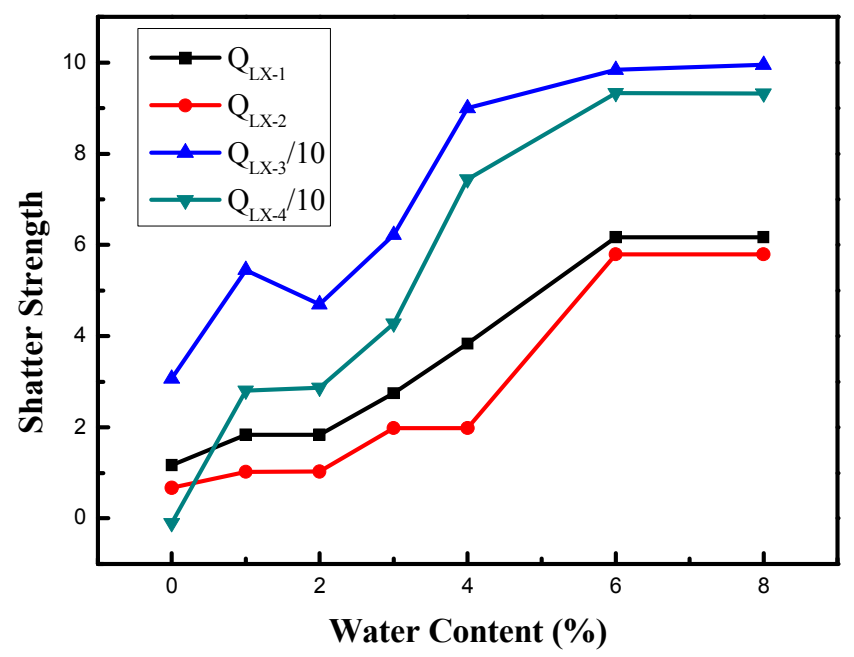

Figure 2. Shatter strength $Q_{L X}$ calculated by different methods.

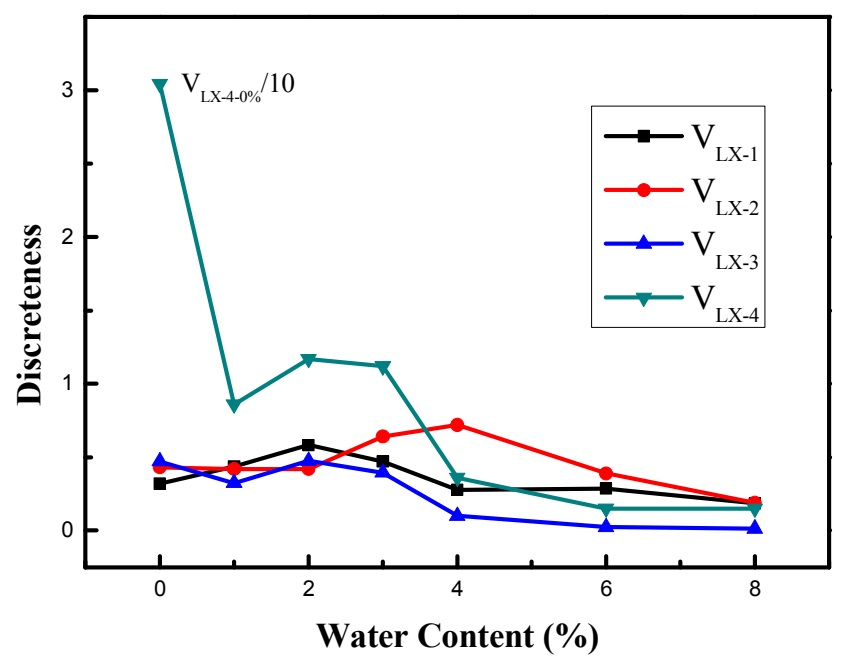

Figure 3. Discreteness $V_{L X}$ of the shatter strength $Q_{L X}$.

The shatter strength $Q_{L X}$ is obtained by different calculation methods, as shown in Figure 2. The trends of the four curves are basically the same when the water content is greater than $4 \%$. The difference between the trends is very large when the water content is $0 \sim 4 \%$. The trends for $Q_{L X-2}$ and $Q_{L X-3}$ are obviously abnormal when the water content is $3 \sim 4 \%$ and $1 \sim 2 \%$, respectively. Because water will make the mineral powder in the pellet pack closer, the shatter strength should be stronger, rather than unchanged or even smaller. Combined with Figure 3, it is found that when the water content is lower, the $V_{L X-i}$ value is greater, indicating that the material property is unstable, which may be caused by the uneven water content in the samples. Although $V_{L X-2}$ and $V_{L X-4}$ are greater than $V_{L X-1}$ and $V_{L X-3}$ when the water content is $2 \sim 6 \%$ and $0 \sim 8 \%, Q_{L X-2}$ and $Q_{L X-4}$ are normal. This shows that the degree of discreteness can reflect the production quality, but it does not affect the evaluation results for the shatter strength. The new methods, Eqs. 1-3 and Eqs. 2-4, take into account the degree of rupture and drop times, increase the influence of these factors on the results, and finally produce results that are in line with the actual situation in the case of a small number of samples.

\section{Conclusions}

(1) Advantages of the new method based on design principles: The new method considers the crack time and degree of rupture (crack size and fragment number), enabling more accurate shatter strength evaluations. The greatest advantage of the new method is that it can distinguish between samples with very small differences in shatter strength and judge the production quality of the whole batch of material through the discreteness $V_{L X}$. Moreover, the parameters $i$ and $k$ of the formula can be adjusted according to different test objectives, enabling a more appropriate range of applicability. Users can also design new methods for all kinds of brittle materials according to these principles.

(2) Disadvantages of the new method: The new method is more complex than the old method and needs to consider a greater number of factors. Therefore, the new method creates a less convenient testing process. Even with automatic dropping devices, the workload is extensive. Furthermore, data processing for the new method is also more difficult than that for the old methods, wherein the former requires software assistance.

(3) Thinking about the design principles. The design principles described in this paper still have shortcomings. First, the design principles are complex and not conducive to being used. Second, the design principles do not consider the influence of the crack depth (a characteristic of the degree of rupture) on the shatter strength. With the development of image recognition technology, this part can be added.

(4) Future prospects of this work: This study did not use coal or coke. To verify the new methods, there may be some shortcomings that need to be resolved. In addition, automatic dropping and judging the degree of rupture are restrictions on limiting the overall speed. Increasing the testing speed is the key to enabling wider use of the new method. At present, automatic lifting/dropping devices are relatively mature, but technology to judge the degree of rupture is still lacking. If machine vision, image recognition technology and automatic screening can be used to judge the degree of rupture, the test can be fully automated and intelligent, thereby greatly improving the speed of testing.

\section{Acknowledgements}

The authors thank the Education Department of Shaanxi Province (No. 18JK0476) for their financial support. 


\section{Appendix}

Collection and Testing: How to Test the Shatter Strength

The data in Table A1-Table A4 were measured and evaluated by the methods in Table 1-No. 1, Eqs. 1-3, Table 1-No. 2 and Eqs. 2-4. The calculation results in Table A1 correspond to the curves in Figures 1 and 2. Notably, $\mathrm{m}_{\mathrm{L}-\mathrm{i}}$ in Eq. 1 is not the same value as $\mathrm{m}_{\mathrm{L}-\mathrm{i}}$ in Eq. 1. The former represents the drop time, and the latter represents the residual mass.

Table A1. Shatter strength of lead-containing pellets measured by the traditional method (Table 1-No. 1).

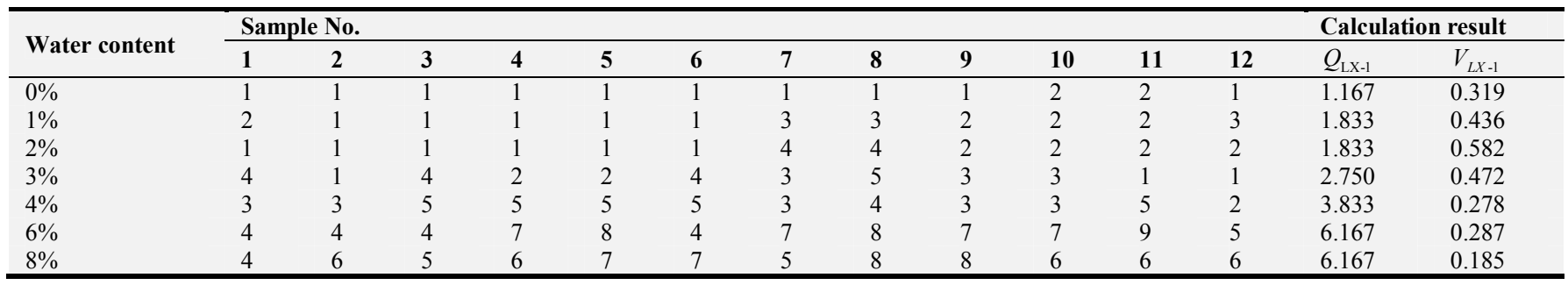

The measured value in Table $\mathrm{A} 1$ is the drop time when cracking or fragmentation occurs. $Q_{L X-1}$ refers to the average of the values in the corresponding row. $V_{L X}$ can be obtained directly according to Eq. 3.

In Table A2, $j$ refers to the number of fragments, which means that the sample has broken into several pieces after fragmentation. $y$ refers to the crack size index, which defines the size of the crack. $m_{L}$ refers to the drop time when cracks are generated. $Q_{L X-i}, Q_{L X-2}$ and $V_{L X-2}$ were calculated by Eqs. 1, 2, 3, respectively.

Table A2. Shatter strength of the lead-containing pellets measured by the new method (Eqs. 1-3).

\begin{tabular}{|c|c|c|c|c|c|c|c|c|c|c|c|c|c|c|c|}
\hline \multirow{2}{*}{ Water content } & \multirow{2}{*}{ Parameter } & \multicolumn{12}{|c|}{ Sample No. } & \multicolumn{2}{|c|}{ Calculation result } \\
\hline & & 1 & 2 & 3 & 4 & 5 & 6 & 7 & 8 & 9 & 10 & 11 & 12 & $Q_{\mathrm{LX}-2}$ & $V_{L X-2}$ \\
\hline \multirow{4}{*}{$0 \%$} & $j$ & 3 & 2 & 1 & 1 & 1 & 1 & 1 & 3 & 3 & 2 & 2 & 3 & \multirow{4}{*}{0.67} & \multirow{4}{*}{0.43} \\
\hline & $y$ & 3 & 3 & 2 & 2 & 2 & 2 & 2 & 3 & 3 & 3 & 3 & 1 & & \\
\hline & $m_{L}$ & 1 & 1 & 1 & 1 & 1 & 1 & 1 & 1 & 1 & 2 & 2 & 1 & & \\
\hline & $Q_{\mathrm{LX}-\mathrm{i}}$ & 0.33 & 0.50 & 1.00 & 1.00 & 1.00 & 1.00 & 1.00 & 0.33 & 0.33 & 0.63 & 0.63 & 0.33 & & \\
\hline \multirow{4}{*}{$1 \%$} & $j$ & 2 & 1 & 2 & 2 & 2 & 1 & 1 & 2 & 1 & 1 & 1 & 1 & \multirow{4}{*}{1.02} & \multirow{4}{*}{0.42} \\
\hline & $y$ & 3 & 1 & 2 & 3 & 3 & 2 & 3 & 3 & 2 & 2 & 2 & 2 & & \\
\hline & $m_{L}$ & 2 & 1 & 1 & 1 & 1 & 1 & 3 & 3 & 2 & 2 & 2 & 3 & & \\
\hline & $Q_{\mathrm{LX}-\mathrm{i}}$ & 0.63 & 1.00 & 0.50 & 0.50 & 0.50 & 1.00 & 1.44 & 0.72 & 1.41 & 1.41 & 1.41 & 1.73 & & \\
\hline \multirow{4}{*}{$2 \%$} & $j$ & 2 & 3 & 1 & 1 & 1 & 1 & 2 & 1 & 2 & 1 & 1 & 1 & \multirow{4}{*}{1.03} & \multirow{4}{*}{0.42} \\
\hline & $y$ & 3 & 3 & 2 & 2 & 1 & 1 & 3 & 2 & 3 & 3 & 2 & 2 & & \\
\hline & $m_{L}$ & 1 & 1 & 1 & 1 & 1 & 1 & 4 & 4 & 2 & 2 & 2 & 2 & & \\
\hline & $Q_{\mathrm{LX}-\mathrm{i}}$ & 0.50 & 0.33 & 1.00 & 1.00 & 1.00 & 1.00 & 0.79 & 2.00 & 0.63 & 1.26 & 1.41 & 1.41 & & \\
\hline \multirow{4}{*}{$3 \%$} & $j$ & 1 & 5 & 1 & 1 & 1 & 1 & 1 & 1 & 1 & 1 & 4 & 2 & \multirow{4}{*}{1.98} & \multirow{4}{*}{0.64} \\
\hline & $y$ & 1 & 3 & 1 & 2 & 2 & 2 & 2 & 2 & 1 & 1 & 3 & 1 & & \\
\hline & $m_{L}$ & 4 & 1 & 4 & 2 & 2 & 4 & 3 & 5 & 3 & 3 & 1 & 1 & & \\
\hline & $Q_{\mathrm{LX}-\mathrm{i}}$ & 4.00 & 0.20 & 4.00 & 1.41 & 1.41 & 2.00 & 1.73 & 2.24 & 3.00 & 3.00 & 0.25 & 0.50 & & \\
\hline \multirow{4}{*}{$4 \%$} & $j$ & 2 & 1 & 1 & 2 & 1 & 1 & 1 & 1 & 2 & 1 & 1 & 2 & \multirow{4}{*}{1.98} & \multirow{4}{*}{0.72} \\
\hline & $y$ & 3 & 3 & 2 & 3 & 1 & 1 & 2 & 3 & 3 & 2 & 3 & 1 & & \\
\hline & $m_{L}$ & 3 & 3 & 5 & 5 & 5 & 5 & 3 & 4 & 3 & 3 & 5 & 2 & & \\
\hline & $Q_{\mathrm{LX}-\mathrm{i}}$ & 0.72 & 1.44 & 2.24 & 0.85 & 5.00 & 5.00 & 1.73 & 1.59 & 0.72 & 1.73 & 1.71 & 1.00 & & \\
\hline \multirow{4}{*}{$6 \%$} & $j$ & 1 & 1 & 1 & 1 & 1 & 1 & 1 & 1 & 1 & 1 & 1 & 2 & \multirow{4}{*}{5.79} & \multirow{4}{*}{0.39} \\
\hline & $y$ & 1 & 1 & 1 & 1 & 1 & 2 & 1 & 1 & 1 & 1 & 1 & 1 & & \\
\hline & $m_{L}$ & 4 & 4 & 4 & 7 & 8 & 4 & 7 & 8 & 7 & 7 & 9 & 5 & & \\
\hline & $Q_{\mathrm{LX}-\mathrm{i}}$ & 4.00 & 4.00 & 4.00 & 7.00 & 8.00 & 2.00 & 7.00 & 8.00 & 7.00 & 7.00 & 9.00 & 2.50 & & \\
\hline \multirow{4}{*}{$8 \%$} & $j$ & 1 & 1 & 1 & 1 & 1 & 1 & 1 & 1 & 1 & 1 & 1 & 1 & \multirow{4}{*}{6.17} & \multirow{4}{*}{0.19} \\
\hline & $y$ & 1 & 1 & 1 & 1 & 1 & 1 & 1 & 1 & 1 & 1 & 1 & 1 & & \\
\hline & $m_{L}$ & 4 & 6 & 5 & 6 & 7 & 7 & 5 & 8 & 8 & 6 & 6 & 6 & & \\
\hline & $Q_{\mathrm{LX}-\mathrm{i}}$ & 4.00 & 6.00 & 5.00 & 6.00 & 7.00 & 7.00 & 5.00 & 8.00 & 8.00 & 6.00 & 6.00 & 6.00 & & \\
\hline
\end{tabular}

The measured values in Table A3 are the residual mass percentage of the samples after the dropping test. $Q_{L X-I}$ is the average of the values in the corresponding row. $V_{L X}$ can be obtained directly according to Eq. 3 .

In Table A4, $j$ refers to the number of fragments. This means that the sample broke into several pieces during fragmentation. $x$ is the drop time when the test is terminated. $m_{0}$ is the original mass of the sample, and $m_{L}$ is the total mass of the fragments conforming to the standard after the test. $Q_{L X-i}, Q_{L X-2}$, and $V_{L X-2}$ were calculated by Eqs. $4,2,3$, respectively. $k$, the total number of drops in Eqs. 4, was 3 in the calculation. 
Table A3. Shatter strength of lead-containing pellets measured by the traditional method (Table 1-No. 2).

\begin{tabular}{|c|c|c|c|c|c|c|c|c|c|c|c|c|c|c|}
\hline \multirow{2}{*}{$\begin{array}{l}\text { Water } \\
\text { content }\end{array}$} & \multicolumn{12}{|c|}{ Sample No. } & \multicolumn{2}{|c|}{ Calculation result } \\
\hline & 1 & 2 & 3 & 4 & 5 & 6 & 7 & 8 & 9 & 10 & 11 & 12 & $\boldsymbol{Q}_{\mathrm{LX}-3}$ & $V_{L X-4}$ \\
\hline $0 \%$ & 12.5 & 27.5 & 52.5 & 37.5 & 40.0 & 26.3 & 21.3 & 12.5 & 15.0 & 58.3 & 40.0 & 25.0 & 30.7 & 0.472 \\
\hline $1 \%$ & 53.8 & 37.5 & 47.5 & 52.5 & 25.0 & 38.3 & 73.3 & 88.3 & 38.0 & 72.0 & 67.5 & 61.0 & 54.5 & 0.324 \\
\hline $2 \%$ & 37.5 & 37.5 & 37.5 & 32.5 & 25.0 & 16.3 & 86.3 & 94.5 & 50.0 & 53.0 & 57.5 & 36.3 & 47.0 & 0.475 \\
\hline $3 \%$ & 87.5 & 25.0 & 87.5 & 50.0 & 62.5 & 89.0 & 55.5 & 85.0 & 41.5 & 93.5 & 24.8 & 45.0 & 62.2 & 0.394 \\
\hline $4 \%$ & 75.3 & 87.5 & 100.0 & 100.0 & 99.8 & 99.5 & 85.3 & 88.0 & 80.0 & 85.0 & 100 & 79.5 & 90.0 & 0.100 \\
\hline $6 \%$ & 95 & 95 & 95 & 100 & 100 & 96 & 99.5 & 99.8 & 100 & 100 & 100 & 100 & 98.4 & 0.023 \\
\hline $8 \%$ & 95.3 & 99.0 & 100.0 & 100 & 100 & 100 & 100 & 100 & 100 & 100 & 100 & 100 & 99.5 & 0.013 \\
\hline
\end{tabular}

Table A4. Shatter strength of the lead-containing pellets measured by the new method (Eqs. 3-4).

\begin{tabular}{|c|c|c|c|c|c|c|c|c|c|c|c|c|c|c|c|}
\hline \multirow{2}{*}{$\begin{array}{l}\text { Water } \\
\text { content }\end{array}$} & \multirow{2}{*}{ Parameter } & \multicolumn{12}{|c|}{ Sample No. } & \multicolumn{2}{|c|}{ Calculation result } \\
\hline & & 1 & 2 & 3 & 4 & 5 & 6 & 7 & 8 & 9 & 10 & 11 & 12 & $Q_{\mathrm{LX}-4}$ & $\mathrm{~V}_{\mathrm{LX}-4}$ \\
\hline \multirow{4}{*}{$0 \%$} & $j$ & 5 & 3 & 2 & 2 & 5 & 1 & 1 & 5 & 5 & 3 & 2 & 5 & & \\
\hline & $m_{0}$ & 2.58 & 2.60 & 2.60 & 2.54 & 2.57 & 2.55 & 2.61 & 2.40 & 2.62 & 2.60 & 2.55 & 2.55 & -1.0 & 30.44 \\
\hline & $m_{L}$ & 0.33 & 0.72 & 1.37 & 0.98 & 1.04 & 0.68 & 0.55 & 0.33 & 0.39 & 1.51 & 1.04 & 0.65 & & \\
\hline & $Q_{\mathrm{LX}-\mathrm{i}}$ & -32 & 9 & 26 & 19 & 8 & 27 & 21 & -66 & -32 & 19 & 20 & -32 & & \\
\hline \multirow{4}{*}{$1 \%$} & $x$ & 3 & 3 & 3 & 3 & 2 & 3 & 3 & 3 & 3 & 3 & 3 & 3 & & \\
\hline & $m_{0}$ & 2.60 & 2.60 & 2.65 & 2.62 & 2.60 & 2.58 & 2.60 & 2.60 & 2.61 & 2.60 & 2.60 & 2.61 & 28.0 & 0.86 \\
\hline & $m_{L}$ & 1.40 & 0.98 & 1.24 & 1.37 & 0.65 & 0.99 & 1.90 & 2.29 & 0.99 & 1.87 & 1.76 & 1.59 & & \\
\hline & $Q_{\mathrm{LX}-\mathrm{i}}$ & 18 & 19 & 23 & 26 & -32 & 13 & 37 & 29 & 38 & 36 & 68 & 61 & & \\
\hline \multirow{4}{*}{$2 \%$} & $j$ & 5 & 5 & 1 & 1 & 2 & 1 & 2 & 1 & 2 & 1 & 1 & 1 & & \\
\hline & $x$ & 2 & 2 & 3 & 3 & 3 & 3 & 3 & 3 & 3 & 3 & 3 & 3 & & \\
\hline & $m_{0}$ & 2.60 & 2.60 & 2.64 & 2.58 & 2.54 & 2.50 & 2.63 & 2.63 & 2.60 & 2.62 & 2.60 & 2.6 & 28.7 & 1.17 \\
\hline & $Q_{\mathrm{LX}-\mathrm{i}}$ & -31 & -31 & 37 & 33 & 13 & 17 & 43 & 93 & 25 & 53 & 58 & 36 & & \\
\hline \multirow{5}{*}{$3 \%$} & $j$ & 1 & 5 & 1 & 2 & 1 & 1 & 1 & 2 & 1 & 1 & 5 & 2 & & \\
\hline & $x$ & 3 & 1 & 3 & 3 & 3 & 3 & 3 & 3 & 3 & 3 & 2 & 3 & & \\
\hline & $m_{0}$ & 2.62 & 2.60 & 2.60 & 2.60 & 2.64 & 2.55 & 2.61 & 2.48 & 2.55 & 2.55 & 2.62 & 2.64 & 42.8 & 1.12 \\
\hline & $m_{L}$ & 2.28 & 0.65 & 2.28 & 1.30 & 1.63 & 2.31 & 1.44 & 2.21 & 1.08 & 2.43 & 0.64 & 1.17 & & \\
\hline & $Q_{\mathrm{LX}-\mathrm{i}}$ & 87 & -66 & 88 & 25 & 62 & 91 & 55 & 45 & 42 & 95 & -32 & 22 & & \\
\hline \multirow{5}{*}{$4 \%$} & $j$ & 2 & 1 & 1 & 3 & 1 & 1 & 1 & 1 & 2 & 1 & 1 & 2 & & \\
\hline & $x$ & 3 & 3 & 3 & 3 & 3 & 3 & 3 & 3 & 3 & 3 & 3 & 3 & & \\
\hline & $m_{0}$ & 2.62 & 2.62 & 2.62 & 2.60 & 2.60 & 2.57 & 2.61 & 2.61 & 2.64 & 2.60 & 2.63 & 2.63 & 74.4 & 0.36 \\
\hline & $m_{L}$ & 1.96 & 2.28 & 2.60 & 2.60 & 2.59 & 2.59 & 2.22 & 2.29 & 2.08 & 2.21 & 2.60 & 2.07 & & \\
\hline & $Q_{\mathrm{LX}-\mathrm{i}}$ & 37 & 87 & 99 & 33 & 100 & 101 & 85 & 88 & 39 & 85 & 99 & 39 & & \\
\hline \multirow[b]{3}{*}{$6 \%$} & $j$ & 1 & 1 & 1 & 1 & 1 & 1 & 1 & 1 & 1 & 1 & 1 & 2 & & \\
\hline & $x$ & 3 & 3 & 3 & 3 & 3 & 3 & 3 & 3 & 3 & 3 & 3 & 3 & & \\
\hline & $m_{0}$ & 2.63 & 2.63 & 2.63 & 2.56 & 2.65 & 2.62 & 2.62 & 2.62 & 2.60 & 2.65 & 2.65 & 2.66 & 93.3 & 0.15 \\
\hline \multirow{5}{*}{$8 \%$} & $j$ & 1.00 & 1.00 & 1.00 & 2.00 & 1.00 & 1.00 & 1.00 & 1.00 & 1.00 & 1.00 & 1.00 & 1 & & \\
\hline & $x$ & 3.00 & 3.00 & 3.00 & 3.00 & 3.00 & 3.00 & 3.00 & 3.00 & 3.00 & 3.00 & 3.00 & 3 & & \\
\hline & $m_{0}$ & 2.66 & 2.66 & 2.67 & 2.70 & 2.67 & 2.62 & 2.64 & 2.64 & 2.65 & 2.65 & 2.69 & 2.7 & 93.2 & 0.15 \\
\hline & $m_{L}$ & 2.48 & 2.57 & 2.60 & 2.60 & 2.60 & 2.60 & 2.60 & 2.60 & 2.60 & 2.60 & 2.60 & 2.60 & & \\
\hline & $Q_{\mathrm{LX}-\mathrm{i}}$ & 93 & 97 & 97 & 48 & 97 & 99 & 98 & 98 & 98 & 98 & 97 & 96 & & \\
\hline
\end{tabular}

\section{References}

[1] Padan J S, Singh O, and Kapoor A N. Raw materials for iron making and their testing. NML Technical Journal, 15 (3). pp. 59-68. http://eprints.nmlindia.org/2141/

[2] Aditya Sarda, Naik Nithesh, Navneeth Krishna, Tarun Shrivastva, and Arun Prabhu. Study and Implementation of Quality Improvement Techniques to Improve the Consistency in Cold Crushing Strength of Iron Ore Pellets. Asian Journal of
Engineering and Applied Technology, 3 (2). pp. 10-15. http://eprints.manipal.edu/147915/

[3] Xiang Feng, Gao De-yun, Zhang Yu-lin, Zhou Liu, Zhang Jia-min, and Fang Shu-ming. On Cold-Pressing Ball of Mn-powder Mine, 2007, 15 (3). pp. 59-68. https://doi.org/10.3969/j.issn.1002-4336.2007.03.008

[4] Cao Ke-ning, He Shan-te Zhang bo-jiang, Fu Chong-Shuo, Chen Guo-fa, Cai Zhi-peng, Xu Hong-zhi, Chen An-ming, and Meng Fan-zhong. Shuikou Mountain Method of Smelt Lead: CN85105034A [P]. 1985-07-03. 
[5] Ying Haisong. Physical inspection and design of contract specifications for iron ore import (J). Metal mine, 2008 (03): 22 -25. https://doi.org/10.3321/j.issn:1001-1250.2008.03.006

[6] FAN Xiao-hui, YUAN Xiao-li, JIANG Tao, YUAN Li-shun, LI Guang-hui, ZHUANG Jian-ming, TANG Qi-rong, and YANG Xue-feng. Effect of particle size distribution of concentrate on palletizing quality $(\mathrm{J})$. The Chinese Journal of Nonferrous Metal, 2006, $16 \quad$ (11): $1965-1970$. https://doi.org/10.3321/j.issn:1004-0609.2006.11.023

[7] Li Yanru, Zhou Mingshun, Zhai Liwei, Zhang Liguo, and Ren Wei. Effect of Types of Bentonites on Green Pellet Property (J).

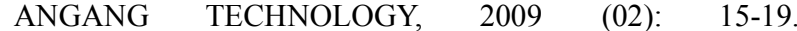
https://doi.org/10.3969/j.issn.1006-4613.2009.02.004

[8] Li Jiaxin, Xiang Dongwen, Wei Rufei, Yang Jialong, Long Hongming, and Yang Lebiao. Method for Detecting Drop Strength of Green Pellets under Motion State: CN201610890303.4 [P]. 2017-05-10.

[9] Jinquan Fan, Huijian Ye. Full-automatic Dropping Strength Test Device: CN200610155181.0 [P]. 2006-12-13.

[10] Li Jiaxin, Xiang Dongwen. Dropping Strength Detection Device and Method of Metallurgical Green Pellets: CN201610613623.5 [P]. 2016-10-12.

[11] JIS M8711-1993, Iron ore sinter -Determination of shatter strength (S). 1993.
[12] FOCT25471-1982, Iron ores, concentrates, agglomerates and pellets. Method for determination of solidity on droption (S). 1982.

[13] KS E3714-1997, Iron ore sinter -Determination of shatter strength (S). 1997/2002.

[14] MT/ T925-2004, Drop shatter test of industrial briquette [S] 2004.

[15] YB/T 4606-2017, Iron and sintering ore -Determination of shatter strength (S). 2017.

[16] GB/T 4511.2-1999, Coke -Determination of shatter strength (S). 1999.

[17] ISO 616-1995, Coke -Determination of shatter indices (S). 1995.

[18] GB/T 15459-2006, Determination of shatter indices of coal (S). 2006.

[19] CHUNG, TAE SIK. Drop Tester for Briquettes to Measure the Strength through Repetitive Drop: KR1020110023974 [P]. 2011-03-09.

[20] Zhang Juan, Zhang Saiping, and Zhang Guanlai. Adjustable drop strength measuring device: CN201410704289 [P]. 2014-11-28. 